Bull. Chem. Soc. Ethiop. 2018, 32(2), 249-257.

ISSN 1011-3924

(c) 2018 Chemical Society of Ethiopia and The Authors

Printed in Ethiopia

DOI: https://dx.doi.org/10.4314/bcse.v32i2.6

\title{
AQUEOUS PHASE SYNTHESIS, CRYSTAL STRUCTURE AND ANTIMICROBIAL ACTIVITY OF 4-(SUBSTITUTED PHENYLAZO)-3-METHYL-4H-ISOXAZOL-5-ONE AZO DYES
}

\author{
Anita R. Banpurkar ${ }^{1 *}$, Sachin S. Wazalwar ${ }^{1}$ and Franc Perdih ${ }^{2}$ \\ ${ }^{1}$ Department of Applied Chemistry, Rajiv Gandhi College of Engineering, Research \& \\ Technology, Chandrapur, MS, India-442403 \\ ${ }^{2}$ Faculty of Chemistry and Chemical Technology, University of Ljubljana, SI-1000 Ljubljana, \\ Slovenia
}

(Received January 3, 2018; Revised February 16, 2018; Accepted June 11, 2018)

\begin{abstract}
Methyl-4H-isoxazol-5-one was synthesized at room temperature by simple stirring method from ethyl acetoacetate and hydroxylamine hydrochloride in aqueous medium and coupled with diazotized substituted amine to form series of 4-(substituted phenylazo)-3-methyl-4H-isoxazol-5-ones through green chemistry. All the compounds formed were characterized by IR, ${ }^{1} \mathrm{H}$ and ${ }^{13} \mathrm{C}$ NMR spectroscopy, MS and elemental analysis. Crystal structure of novel 4-(4-fluorophenylazo)-3-methyl-4H-isoxazol-5-one was determined by the X-ray diffraction. Antibacterial and antifungal activity was studied against Escherichia coli, Pseudomonas aeruginosa, Staphylococcus aureus, Staphylococcus pyogenus and Candida albicans, Aspergillus niger, Aspergillus clavatus, respectively. All synthesized compounds were found to be active against a gram-positive bacterium Staphylococcus aureus. Two compounds showed antifungal activity against Candida albicans close to standard greseofulvin.
\end{abstract}

KEY WORDS: Azo dyes, Substituted amines, Antibacterial and antifungal activity, X-ray diffraction, Spectroscopy, Green chemistry

\section{INTRODUCTION}

Azo dyes are the most widely used class of coloring materials because of their fruitful applications in various fields of science and technology [1-3] and also for their interesting structural and physicochemical properties [4-6]. Azo dyes are synthesized by diazotization of aromatic amines and coupling reagent, which include one or more azo groups $(-\mathrm{N}=\mathrm{N}-)$ attached to one or more aromatic moieties [7]. Currently, heteroarylazoisoxazol compounds have received the attention of many research groups [8] because of their wide spread potential applications in fields of catalysis [9], cancer treatment and as antibacterial and antiviral agents, agricultural fungicides as well as other biological uses [10-12]. Intramolecular $\mathrm{N}-\mathrm{H} \cdots \mathrm{O}$ hydrogen bonding study assisted by resonance for 1-ketone-2-arylhydrazones derivatives suggest that heterocyclic five member ring introduces geometrical constraint which hinders the strengthening of hydrogen bond [13]. Tautomerism and spectroscopic properties of some heteroarylazoisoxazolone dyes were studied earlier [14]. With these objects in view we focus on the synthesis and antimicrobial activity of 4-(substituted phenylazo)-3-methyl-4H-isoxazol-5one azo dyes.

\section{EXPERIMENTAL}

All the starting materials and solvents were purchased from commercial sources and were used without further purification. Melting points were determined in open capillaries using electro thermal melting point apparatus and are uncorrected. Progress of reactions was monitored by TLC. Infrared (IR) spectra $\left(4000-600 \mathrm{~cm}^{-1}\right)$ of the samples were recorded using a Perkin-Elmer Spectrum 100, equipped with a Specac Golden Gate Diamond ATR as a solid sample support.

*Corresponding author. E-mail: anitabanpurkar1@gmail.com

This work is licensed under the Creative Commons Attribution 4.0 International License 
${ }^{1} \mathrm{H}$ NMR spectra were recorded with a Bruker Avance III 500 nuclear magnetic resonance spectrometer with TMS as internal reference. ${ }^{13} \mathrm{C}$ NMR spectra were recorded with a Bruker Avance III 500 spectrometer at $125 \mathrm{MHz}$ and were referenced against the central line of the solvent signal $\left(\mathrm{CDCl}_{3}\right.$ triplet at $77.0 \mathrm{ppm}$ or DMSO- $d_{6}$ septet at $\left.39.5 \mathrm{ppm}\right)$. MS spectra were recorded with an Agilent 6624 Accurate Mass TOF LC/MS instrument (ESI ionization).

Single-crystal X-ray diffraction data for $\mathbf{6 c} \cdot 0.3 \mathrm{H}_{2} \mathrm{O}$ were collected on an Agilent Technologies SuperNova Dual diffractometer with the Atlas detector using monochromated Mo-K $\alpha$ radiation $(\lambda=0.71073 \AA)$ at room temperature. The data were processed using CrysAlis Pro. [15]. The structure was solved by direct methods using the program Superflip [16] and refined on $F^{2}$ using full-matrix least-squares procedures using SHELXL2014 [17]. All nonhydrogen atoms were refined anisotropically. Hydrogen atoms were readily located in a difference Fourier maps and were subsequently treated as riding atoms in geometrically idealized positions, with $\mathrm{C}-\mathrm{H}=0.93$ (aromatic) or $0.96 \AA\left(\mathrm{CH}_{3}\right), \mathrm{N}-\mathrm{H}=0.86 \AA$ and with $U_{\text {iso }}(\mathrm{H})=k U_{\text {eq }}(\mathrm{C}$ or $\mathrm{N})$, where $k=1.5$ for methyl groups, which were permitted to rotate but not to tilt, and 1.2 for all other $\mathrm{H}$ atoms. The water $\mathrm{H}$-atoms were refined with a distance restraint and with $U_{\text {iso }}(\mathrm{H})=1.5 U_{\text {eq }}(\mathrm{O})$. Initially, the occupation factor for a water solvate molecule was refined, but was set as fixed at 0.30 in the final stage of the refinement. Structure was refined as a twin. Crystallographic data are listed in Table 1.

Determination of minimal inhibition concentrations by micro broth dilution for antibacterial and antifungal activity

All the synthesized drugs were used for antibacterial test procedures. All necessary controls like drug control, vehicle control, agar control, organism control, known antibacterial drugs control, all MTCC cultures were tested against above mentioned known and unknown drugs. Mueller Hinton broth was used as nutrient medium to grow and dilute the drug suspension for the test bacteria. Inoculum size for test strain was adjusted to $10^{8} \mathrm{Cfu}$ [Colony Forming Unit] per milliliter by comparing the turbidity. Common standard strains were used for screening of antibacterial and antifungal activities: The strains were procured from Institute of Microbial Technology, Chandigarh: E. coli (MTCC443), P. aeruginosa (MTCC1688), S. aureus (MTCC96), S. pyogenus (MTCC442), C. albicans (MTCC227), A. niger (MTCC282), A. clavatus (MTCC1323). DMSO was used as diluents / vehicle to get desired concentration of drugs to test upon standard bacterial strains.

The main advantage of the 'Broth Dilution Method' for minimal inhibition concentration (MIC) determination lies in the fact that it can readily be converted to determine the MIC as well [18-22]. Serial dilutions were prepared in primary and secondary screening. The control tube containing no antibiotic is immediately sub cultured (before inoculation) by spreading a loopful evenly over a quarter of plate of medium suitable for the growth of the test organism and put for incubation at $37{ }^{\circ} \mathrm{C}$ overnight. The tubes are then incubated overnight. The MIC of the control organism is read to check the accuracy of the drug concentrations. The lowest concentration inhibiting growth of the organism is recorded as the MIC. The amount of growth from the control tube before incubation is compared [23].

Each synthesized drug was diluted obtaining $2000 \mu \mathrm{g} / \mathrm{mL}$ concentration, as a stock solution. In primary screening 1000,500 , and $250 \mu \mathrm{g} / \mathrm{mL}$ concentrations of the synthesized drugs were taken. The active synthesized drugs found in this primary screening were further tested in a second set of dilution against all microorganisms. The drugs found active in primary screening were similarly diluted to obtain $200,100,50,25,12.5,6.250 \mu \mathrm{g} / \mathrm{mL}$ end concentrations. The highest dilution showing at least $99 \%$ inhibition zone is taken as MIC. 
General procedure for synthesis of 3-methyl-4-H-isoxazol-5-one

Hydroxylamine hydrochloride $(1.041 \mathrm{~g}, 15 \mathrm{mmol})$ was dissolved in $20 \mathrm{~mL}$ of water and cooled to about $10{ }^{\circ} \mathrm{C}$, added ethyl acetoacetate $(1.95 \mathrm{~g}, 15 \mathrm{mmol})$. The mixture was shaken thoroughly in absence of any catalyst till the appearance of pale yellow color solution of 3-methyl- $4 \mathrm{H}$ isoxazol-5-one [24].

General procedure for synthesis of 4-(substituted phenylazo)-3-methyl-4H-isoxazol-5-one azo dyes

Substituted amine 4a-e $(15 \mathrm{mmol})$ were dissolved in dilute $\mathrm{HCl}(1.5 \mathrm{~mL}$ in $15 \mathrm{~mL}$ water $)$ and cooled in an ice bath to $0-5{ }^{\circ} \mathrm{C}$. The reaction mixture was added to the solution of sodium nitrite $\left(1.043 \mathrm{~g}, 15 \mathrm{mmol}\right.$ in $15 \mathrm{~mL}$ of water) and $0.01 \mathrm{M} \mathrm{HCl}(15 \mathrm{~mL})$ at about $0-5{ }^{\circ} \mathrm{C}$ with continuous stirring to give yellow colored solution 5a-e. To this solution, equimolar amount of cooled 3methyl- $4 H$-isoxazol-5-one solution was added in ice bath with continuous stirring for 30 min to get yellow orange colored precipitate. The $\mathrm{pH}$ of reaction mixture was maintained between 5 and 6 . The reaction mixture was kept overnight at about $15^{\circ} \mathrm{C}$ for the completion of the reaction. The precipitate obtained $\mathbf{6 a}-\mathbf{e}$ was washed with cold water, dried and recrystallized using ethanol. Crystals of $\mathbf{6 c}$ suitable for single crystal XRD analysis were obtained by slow evaporation of ethanol and chloroform $(1: 1 \mathrm{v} / \mathrm{v})$ solvent mixture.

\section{Spectral data}

3-Methyl-4-phenylazo-4H-isoxazol-5-one (6a). Yellow solid, yield: $2.45 \mathrm{~g} \mathrm{(85 \% ),} \mathrm{m.p.:} 170{ }^{\circ} \mathrm{C}$,

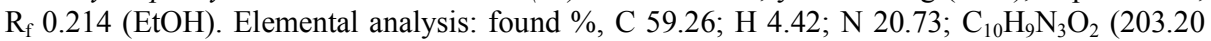
$\mathrm{g} / \mathrm{mol}$ ). Calculated, \% C 59.11; H 4.46; N 20.68;MS (ESI+) $m / z: 204.0767\left(\mathrm{MH}^{+}\right)$, HRMS: found, $m / z$ : 204.0768; $\mathrm{C}_{10} \mathrm{H}_{9} \mathrm{~N}_{3} \mathrm{O}_{2}$ calculated, $\mathrm{m} / z$ : 204.0767. IR $\left(\mathrm{cm}^{-1}\right): 3209(\mathrm{NH}), 1708(\mathrm{C}=\mathrm{O})$, $1570(\mathrm{CH}=\mathrm{N}), 1482(\mathrm{C}=\mathrm{C}), 1277(\mathrm{C}-\mathrm{O}) ;{ }^{1} \mathrm{H}$ NMR $\left(500 \mathrm{MHz}, \mathrm{CDCl}_{3}, \delta \mathrm{ppm}\right): 2.26(\mathrm{~s}, 3 \mathrm{H}$, 3- $\left.\mathrm{CH}_{3}\right), 7.37-7.19(\mathrm{~m}, 5 \mathrm{H}, \mathrm{Ar}-\mathrm{H}), 12.60(\mathrm{~s}, 1 \mathrm{H},-\mathrm{NH}) ;{ }^{13} \mathrm{C} \mathrm{NMR}, \delta \mathrm{ppm}, \mathrm{CDCl}_{3}: 10.3,116.1$, $121.1,126.7,129.8,140.4,159.4,164.9$.

4-(4-Chloro-phenylazo)-3-methyl-4H-isoxazol-5-one (6b). Yellow solid, yield: $2.79 \mathrm{~g}$ (82\%), m.p.: $155^{\circ} \mathrm{C}, \mathrm{R}_{\mathrm{f}} 0.224$ (EtOH). Elemental analysis: found \%, C 50.57; H 3.47; N 17.31; $\mathrm{C}_{10} \mathrm{H}_{8} \mathrm{ClN}_{3} \mathrm{O}_{2}$ (237.64 g/mol). Calculated, \% C 50.54; $\mathrm{H}$ 3.39; N 17.68. MS (ESI + ) $\mathrm{m} / \mathrm{z}$ : $238.0377\left(\mathrm{MH}^{+}\right)$, HRMS: found, $m / z$ : 238.0378; $\mathrm{C}_{10} \mathrm{H}_{9} \mathrm{~N}_{3} \mathrm{O}_{2}$ calculated, $m / z: 238.0377$. IR $\left(\mathrm{cm}^{-}\right.$ $\left.{ }^{1}\right): 3204(\mathrm{NH}), 1739(\mathrm{C}=\mathrm{O}), 1554(\mathrm{CH}=\mathrm{N}), 1477(\mathrm{C}=\mathrm{C}), 1220(\mathrm{C}-\mathrm{O}) .{ }^{1} \mathrm{H}$ NMR $(500 \mathrm{MHz}$, $\left.\mathrm{CDCl}_{3}, \delta \mathrm{ppm}\right): 2.26\left(\mathrm{~s}, 3 \mathrm{H},-\mathrm{CH}_{3}\right.$ isoxazol), 7.35-7.28 (m, 4H, $\left.\mathrm{Ar}-\mathrm{H}\right), 12.57(\mathrm{~s}, 1 \mathrm{H},-\mathrm{NH}),{ }^{13} \mathrm{C}$ NMR (125 MHz, $\left.\mathrm{CDCl}_{3}, \delta \mathrm{ppm}\right): 10.3,117.2,130.0,132.0,139.0,159.3,164.8$.

4-(4-Fluorophenylazo)-3-methyl-4H-isoxazol-5-one (6c). Orange yellow solid, yield: $2.60 \mathrm{~g}$ (83\%), m.p.: $160^{\circ} \mathrm{C}, \mathrm{R}_{\mathrm{f}} 0.217(\mathrm{EtOH})$. Elemental analysis: found \%, C 54.60; H 3.25; N 18.80; $\mathrm{C}_{10} \mathrm{H}_{8} \mathrm{FN}_{3} \mathrm{O}_{2}$ (221.19 g/mol). Calculated, \% C 54.30; H 3.65; N 19.00. MS, (ESI + ) $\mathrm{m} / \mathrm{z}$ : 222.0671 $\left(\mathrm{MH}^{+}\right)$, HRMS: found, $m / z: 222.0673 ; \mathrm{C}_{10} \mathrm{H}_{8} \mathrm{FN}_{3} \mathrm{O}_{2}$ calculated, $m / z: 222.0671$. IR $\left(\mathrm{cm}^{-1}\right): 3199(\mathrm{NH}), 1714(\mathrm{C}=\mathrm{O}), 1565(\mathrm{CH}=\mathrm{N}), 1493(\mathrm{C}=\mathrm{C}), 1215(\mathrm{C}-\mathrm{O}) .{ }^{1} \mathrm{H}$ NMR $(500 \mathrm{MHz}$, $\left.\mathrm{CDCl}_{3}, \delta \mathrm{ppm}\right): 2.26$ (s, 3H, $-\mathrm{CH}_{3}$ isoxazol), 7.09-7.06 (m, 2H, Ar-H), 7.35-7.32 (m, 2H, Ar-H), $12.62(\mathrm{~s}, 1 \mathrm{H},-\mathrm{NH}) .{ }^{13} \mathrm{C} \mathrm{NMR}\left(125 \mathrm{MHz}, \mathrm{CDCl}_{3}, \delta \mathrm{ppm}\right): 10.2,116.7,136.7,159.3,160.1$, $162.1,164.0$.

4-(3-Methyl-5-oxo-4,5-dihydroisoxazol-4-ylazo)benzenesulfonamide (6d). Orange yellow solid, yield 3.20 g (79\%), m.p. $240{ }^{\circ} \mathrm{C}, \mathrm{R}_{\mathrm{f}} 0.260(\mathrm{EtOH})$. Elemental analysis found, \%: C 42.40; $\mathrm{H}$ 3.41; N 19.40; $\mathrm{C}_{10} \mathrm{H}_{10} \mathrm{~N}_{4} \mathrm{O}_{4} \mathrm{~S}(282.28 \mathrm{~g} / \mathrm{mol})$. Calculated, \% C 42.55; H 3.57; N, 19.85.MS, (ESI+) $m / z: 283.0491\left(\mathrm{MH}^{+}\right)$, HRMS: found, $m / z: 283.0495 ; \mathrm{C}_{10} \mathrm{H}_{10} \mathrm{~N}_{4} \mathrm{O}_{4} \mathrm{~S}$ calculated, $m / z$ : 
283.0491. IR $\left(\mathrm{cm}^{-1}\right): 3317(\mathrm{NH}), 1719(\mathrm{C}=\mathrm{O}), 1570(\mathrm{CH}=\mathrm{N}), 1482(\mathrm{C}=\mathrm{C}), 1246(\mathrm{C}-\mathrm{O}) .{ }^{1} \mathrm{H}$ NMR (500 MHz, $\left.\mathrm{CDCl}_{3}, \delta \mathrm{ppm}\right): 2.29\left(\mathrm{~s}, 3 \mathrm{H},-\mathrm{CH}_{3}\right.$ isoxazol), $4.71\left(\mathrm{~s}, 2 \mathrm{H},-\mathrm{NH}_{2}\right), 7.46-7.44(\mathrm{~d}$, $2 \mathrm{H}, \mathrm{Ar}-\mathrm{H}), 7.94-7.92$ (d, 2H, Ar-H), 12.56 (s, 1H, -NH). ${ }^{13} \mathrm{C}$ NMR (125 MHz, DMSO, $\delta$ ppm): $10.6,117.2,122.7,127.5,141.2,144.4,160.5,162.4$.

4-(3-Acetylphenylazo)-3-methyl-4H-isoxazol-5-one (6e). Orange solid, yield: $2.76 \mathrm{~g}(79 \%)$, m.p.: $210{ }^{\circ} \mathrm{C}, \mathrm{R}_{\mathrm{f}} 0.251$ (EtOH). Elemental analysis found, \%: C 59.42; H 3.20; N 16.77; $\mathrm{C}_{12} \mathrm{H}_{11} \mathrm{~N}_{3} \mathrm{O}_{3}(245.23 \mathrm{~g} / \mathrm{mol})$. Calculated, \% C 58.77; $\mathrm{H}$ 4.52; N 17.17, MS, (ESI+) $\mathrm{m} / \mathrm{z}$ : 246.0871 $\left(\mathrm{MH}^{+}\right)$, HRMS: found, $m / z: 246.0873 ; \mathrm{C}_{12} \mathrm{H}_{11} \mathrm{~N}_{3} \mathrm{O}_{3}$ calculated, $m / z: 246.0871$. IR $\left(\mathrm{cm}^{-1}\right)$ : $3204(\mathrm{NH}), 1734(\mathrm{C}=\mathrm{O}), 1549(\mathrm{CH}=\mathrm{N}), 1431(\mathrm{C}=\mathrm{C}), 1272(\mathrm{C}-\mathrm{O}) .{ }^{1} \mathrm{H}$ NMR $(500 \mathrm{MHz}$, $\left.\mathrm{CDCl}_{3}, \delta \mathrm{ppm}\right): 2.29\left(\mathrm{~s}, 3 \mathrm{H},-\mathrm{CH}_{3}\right.$ isoxazol), $2.51\left(\mathrm{~s}, 3 \mathrm{H},-\mathrm{COCH}_{3}\right), 7.92-7.47(\mathrm{~m}, 4 \mathrm{H}, \mathrm{Ar}-\mathrm{H})$, 12.63 (s, $1 \mathrm{H},-\mathrm{NH}) .{ }^{13} \mathrm{C} \mathrm{NMR}\left(125 \mathrm{MHz}, \mathrm{CDCl}_{3}, \delta \mathrm{ppm}\right): 10.3,26.7,115.3,120.2,126.3,130.2$, $138.6,140.9,159.4,164.7,196.9$.

\section{RESULTS AND DISCUSSION}

A series of 4-(substituted phenylazo)-3-methyl-4- $H$-isoxazol-5-one azo dyes were obtained from easily available, economically feasible chemicals like ethyl acetoacetate, hydroxylamine hydrochloride and some substituted amines by environmentally benign, green route in aqueous medium without using any catalyst. In the first step the reaction of ethyl acetoacetate (1) and hydroxylamine hydrochloride (2) resulted in the formation of oxime, which on further ring closure formed 3-methyl-4H-isoxazol-5-one (3) (Scheme 1). Electron withdrawing effect of $\mathrm{N}$ atom in 3-methyl-4H-isoxazol-5-one along with electron donating effect of $\mathrm{O}$ atom and presence of electron releasing methyl and keto groups makes it more reactive [15]. In the second step diazotization of substituted amine 4a-e was carried out in the presence of sodium nitrite and hydrochloric acidwhich then coupled with active hydrogen atom of isoxazol nucleus under cold condition to give desired product (Scheme 1).

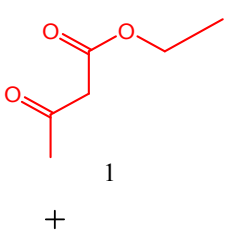

$\mathrm{NH}_{2} \mathrm{OH} . \mathrm{HCl}$

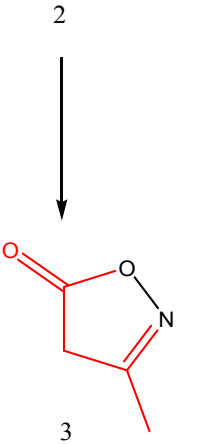<smiles>Nc1ccccc1</smiles>

$4(\mathrm{a}-\mathrm{e})$

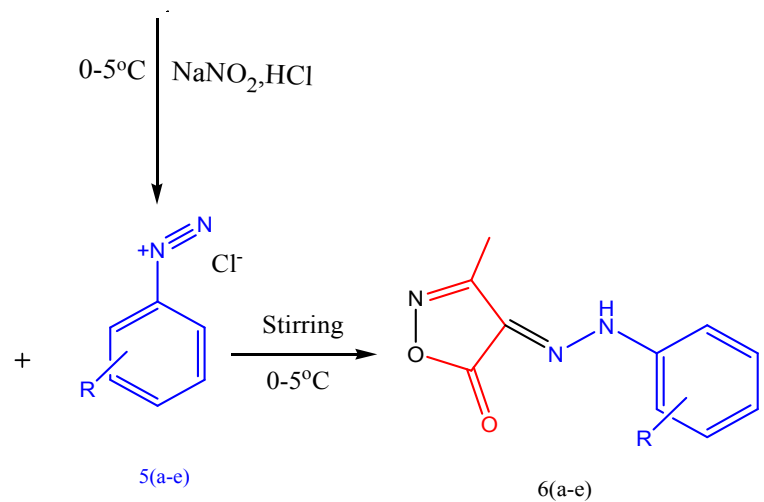

a: $\mathrm{R}=\mathrm{H}, \mathrm{b}: \mathrm{R}=4-\mathrm{Cl}$, c: $\mathrm{R}=4-\mathrm{F}, \mathrm{d}: \mathrm{R}=4-\mathrm{SO}_{2} \mathrm{NH}_{2}, \mathrm{e}: \mathrm{R}=3-\mathrm{COCH}_{3}$

Scheme 1: Synthesis of 4-(substituted phenylazo)-3-methyl-4H-isoxazol-5-one azo dyes.

Bull. Chem. Soc. Ethiop. 2018, 32(2) 
All compounds 6a-e show singlet for NH proton in the range of $12.50-12.60 \mathrm{ppm}$. Multiplets for aromatic ring were observed between 7-8 ppm. A singlet for three protons in the range of 2.26-2.29 is attributed to $\mathrm{CH}_{3}$ group of isoxazol. Compound 6d shows a singlet for two protons of $\mathrm{NH}_{2}$ group related to sulphanilamide moiety at $4.71 \mathrm{ppm}$ and compound 6e show a singlet for three protons related to $\mathrm{COCH}_{3}$ group at $2.51 \mathrm{ppm} .{ }^{13} \mathrm{C}$ NMR study of $\mathbf{6 a}-\mathbf{e}$ shows a singlet at about $10.24-10.56 \mathrm{ppm}$ for $\mathrm{CH}_{3}$ group of isoxazol, about $160-165 \mathrm{ppm}$ for $\mathrm{C}=\mathrm{O}$ group of isoxazol, compound $\mathbf{6 e}$ shows singlet at $197 \mathrm{ppm}$ for $\mathrm{C}=\mathrm{O}$ group of acetophenone and at 26 ppm for $\mathrm{CH}_{3}$ group of acetophenone. The IR spectrum of compound $\mathbf{6 a - e}$ showed characteristic $v(-\mathrm{NH})$ absorption band in the region 3024-3317 $\mathrm{cm}^{-1}, v(\mathrm{C}=\mathrm{O})$ absorption band in the region $1708-1739 \mathrm{~cm}^{-1}$ and $v(\mathrm{CH}=\mathrm{N})$ absorption band in the region $1550-1570 \mathrm{~cm}^{-1}$ (Figure 1).

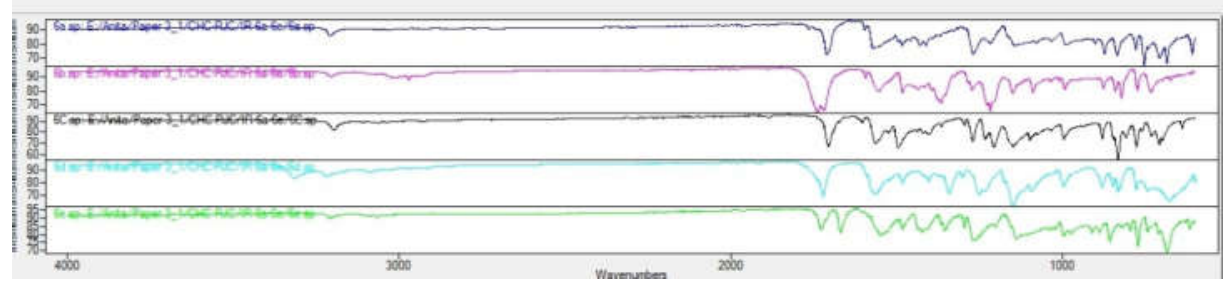

Figure 1. IR spectra of $\mathbf{6 a}-\mathbf{e}$.

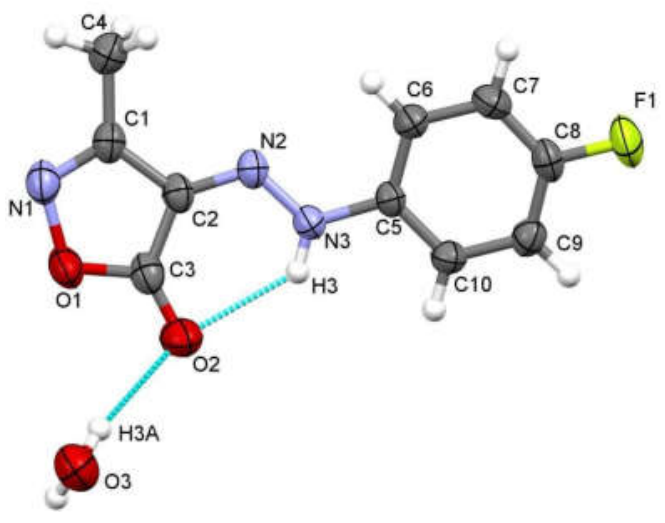

Figure 2. Molecular structure and atom numbering scheme for 6c. Elipsoid Probability are drawn at the $50 \%$ level and hydrogen bonding of $\mathrm{C} 4-\mathrm{H} 4 \mathrm{~A} \cdots \mathrm{O} 2$ and $\mathrm{C} 10-\mathrm{H} 10 \cdots \mathrm{O} 1$ is indicated by blue dashed lines.

Single-crystal X-ray structures of 4-(4-fluorophenylazo)-3-methyl-4H-isoxazol-5-one $\left(\mathbf{6 c} \cdot 0.3 \mathrm{H}_{2} \mathrm{O}\right.$ ) was determined (Figure 2, Table 1). Table 3 and 4 describes significant bond distances and bond angles. All the bond lengths and bond angles of compounds are within normal ranges for this type of structure as reported earlier [13]. Molecule 6c is almost planar, maximum deviation from the mean plane described by all atoms of $\mathbf{6} \mathbf{c}$ is -0.132 (3) $\AA$ for atom $\mathrm{C} 10$. The dihedral angle between the isoxazolone and phenyl rings is $7.81(18)^{\circ}$. In the crystal structure bond distances indicates that the isoxazolone ring contains one double bond, i.e. C1-N1. Also, atoms $\mathrm{C} 2$ and N2 are connected through double bond and intramolecular $\mathrm{N} 3-\mathrm{H} 3 \cdots \mathrm{O} 2$ hydrogen bonding is present between $\mathrm{NH}$ group and the carbonyl group of the isoxazole ring. A water solvate molecule present in the crystal lattice with $30 \%$ occupancy 
forms $\mathrm{O} 3-\mathrm{H} 3 \mathrm{~A} \cdots \mathrm{O} 2$ hydrogen bond with two adjacent molecules of $\mathbf{6 c}$. Furthermore, molecules are linked into chains via $\mathrm{C} 4-\mathrm{H} 4 \mathrm{~A} \cdots \mathrm{O} 2$ interactions between methyl group of isoxazole ring and carbonyl oxygen atom of isoxazole moiety of the adjacent molecule and $\mathrm{C} 10-\mathrm{H} 10 \cdots \mathrm{O} 1$ interactions between the phenyl moiety and the oxygen atom of isoxazole moiety of the adjacent molecule (Figure 3).

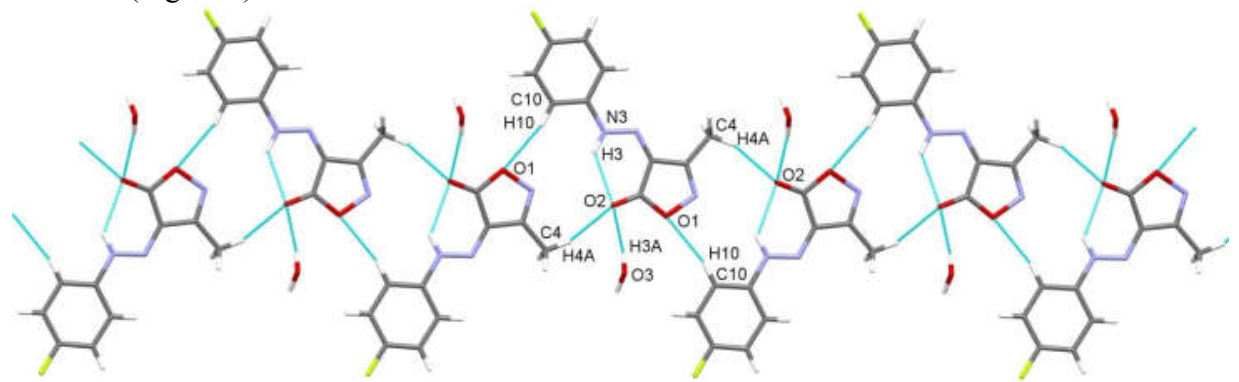

Figure 3. Chain formation generated by $\mathrm{C} 4-\mathrm{H} 4 \mathrm{~A} \cdots \mathrm{O} 2$ and $\mathrm{C} 10-\mathrm{H} 10 \cdots \mathrm{O} 1$ interactions. Hydrogen-bonds are indicated by blue dashed lines.

Table 1. Crystallographic data for 4-(4-fluorophenylazo)-3-methyl- $4 \mathrm{H}$-isoxazol-5-one $\left(\mathbf{6 c} \cdot 0.3 \mathrm{H}_{2} \mathrm{O}\right)$.

\begin{tabular}{|c|c|}
\hline Compound code & 6c. $0.3 \mathrm{H}_{2} \mathrm{O}$ \\
\hline CCDC number & 1561281 \\
\hline Molecular formula & $\mathrm{C}_{10} \mathrm{H}_{8.6} \mathrm{FN}_{3} \mathrm{O}_{2.3}$ \\
\hline Molecular weight & 226.60 \\
\hline Crystal System & Orthorhombic \\
\hline Space group & $F d d 2$ \\
\hline \multicolumn{2}{|l|}{ Unit cell dimensions $(\AA)$} \\
\hline$a$ & $24.2754(17)$ \\
\hline$b$ & $24.5121(17)$ \\
\hline$c$ & $6.8469(5)$ \\
\hline$Z$ & 16 \\
\hline$V\left(\AA^{3}\right)$ & $4074.2(5)$ \\
\hline$D_{\text {calc }}\left(\mathrm{g} \mathrm{cm}^{-3}\right)$ & 1.478 \\
\hline$\mu\left(\mathrm{mm}^{-1}\right)$ & 0.120 \\
\hline$F(000)$ & 1872 \\
\hline Reflections collected & 8741 \\
\hline Parameters & 155 \\
\hline Goodness-of-fit on $F^{2}$ & 1.069 \\
\hline$R_{1}, w R_{2}[I>2 \sigma(I)]^{a}$ & $0.0407,0.0939$ \\
\hline$R_{1}, w R_{2}$ (all data) $)^{b}$ & $0.0560,0.1023$ \\
\hline$\Delta \rho_{\min }, \Delta \rho_{\max }\left(\mathrm{e} \AA^{-3}\right)$ & $-0.172,0.13$ \\
\hline
\end{tabular}

Table 2. Hydrogen bond geometry of $\mathbf{6 c}\left(\AA\right.$ and $\left.^{\circ}\right)$.

\begin{tabular}{|l|l|l|l|l|l|}
\hline $\mathrm{D}-\mathrm{H} \cdots \mathrm{A}$ & $\mathrm{D}-\mathrm{H}(\AA)$ & $\mathrm{H} \cdots \mathrm{A}(\AA)$ & $\mathrm{D} \cdots \mathrm{A}(\AA)$ & $\mathrm{D}-\mathrm{H} \cdots \mathrm{A}\left({ }^{\circ}\right)$ & Symmetry code \\
\hline $\mathrm{N} 3-\mathrm{H} 3 \cdots \mathrm{O} 2$ & 0.86 & 2.23 & $2.882(3)$ & 132.4 & $x, y, z$ \\
\hline $\mathrm{O} 3-\mathrm{H} 3 \mathrm{~A} \cdots \mathrm{O} 2$ & $0.82(2)$ & $2.20(3)$ & $3.007(4)$ & $169(8)$ & $x, y, z$ \\
\hline $\mathrm{C} 4-\mathrm{H} 4 \mathrm{~A} \cdots \mathrm{O} 2$ & 0.96 & 2.56 & $3.371(4)$ & 142.8 & $-x+3 / 4, y-1 / 4, z+1 / 4$ \\
\hline $\mathrm{C} 10-\mathrm{H} 10 \cdots \mathrm{O} 1$ & 0.93 & 2.55 & $3.379(3)$ & 149.4 & $-x+3 / 4, y+1 / 4, z-1 / 4$ \\
\hline
\end{tabular}


Table 3. Significant bond distances.

\begin{tabular}{|c|c|c|}
\hline Sr. No. & Bond & Distance $(\AA)$ \\
\hline 1 & F1 C8 & $1.363(3)$ \\
\hline 2 & O1 C3 & $1.359(3)$ \\
\hline 3 & O1 N1 & $1.470(3)$ \\
\hline 4 & O2 C3 & $1.213(4)$ \\
\hline 5 & N1 C1 & $1.292(3)$ \\
\hline 6 & N2 N3 & $1.306(3)$ \\
\hline 7 & N2 C2 & $1.311(3)$ \\
\hline 8 & N3 C5 & $1.412(3)$ \\
\hline
\end{tabular}

Table 4. Significant bond angles.

\begin{tabular}{|c|c|c|}
\hline Sr. No. & Bond & Angle ( ${ }^{\circ}$ \\
\hline 1 & C3 O1 N1 & $109.2(2)$ \\
\hline 2 & C1 N1 O1 & $106.6(2)$ \\
\hline 3 & N3 N2 C2 & $118.4(2)$ \\
\hline 4 & N2 N3 C5 & $120.0(2)$ \\
\hline 5 & N1 C1 C2 & $11.9(3)$ \\
\hline 6 & N1 C1 C4 & $120.9(3)$ \\
\hline 7 & C2 C1 C4 & $127.2(2)$ \\
\hline 8 & N2 C2 C1 & $125.2(2)$ \\
\hline 9 & N2 C2 C3 & $105.1(2)$ \\
\hline 10 & C1 C2 C3 & $122.1(3)$ \\
\hline 11 & O2 C3 O1 & $131.1(3)$ \\
\hline 12 & O2 C3 C2 & $106.7(2)$ \\
\hline 13 & O1 C3 C2 & \\
\hline
\end{tabular}

All synthesized compounds 6a-e were screened for antibacterial and antifungal study against E. coli, P. aeruginosa, S. aureus and S. pyogenus as bacterial strain and C. albicans, $A$. niger, A. clavatus as fungal strain. All synthesized compounds 6 a-e showed antibacterial activity against $S$. aureus. Compound $\mathbf{6 c}$ and $\mathbf{6 e}$ showed antibacterial activity against $E$. coli whereas compound $\mathbf{6 b}$ showed antibacterial activity against $S$. pyogenus close to activity of standard ampicillin (Table 5). It was found that compounds $\mathbf{6 b}$ and $\mathbf{6 e}$ show antifungal activity against $C$. albicans close to standard greseofulvin (Table 6).

Table 5 . Antibacterial activity study of $\mathbf{6 a}-\mathbf{e}$ with standard drugs.

\begin{tabular}{|l|l|l|l|l|l|}
\hline \multicolumn{7}{|c|}{ Minimal inhibition concentration $(\mu \mathrm{g} / \mathrm{mL})$} \\
\hline Entry & Compound & $\begin{array}{l}\text { E. coli } \\
\text { (MTCC443) }\end{array}$ & $\begin{array}{l}\text { P. aeruginosa } \\
\text { (MTCC1688) }\end{array}$ & $\begin{array}{l}\text { S. aureus } \\
\text { (MTCC96) }\end{array}$ & $\begin{array}{l}\text { S. pyogenus } \\
\text { (MTCC442) }\end{array}$ \\
\hline 1 & 6a & 125 & 200 & 100 & 125 \\
\hline 2 & 6b & 200 & 500 & 200 & 100 \\
\hline 3 & 6c & 62.5 & 250 & 200 & 200 \\
\hline 4 & 6d & 200 & 250 & 100 & 200 \\
\hline 5 & 6e & 100 & 250 & 250 & 250 \\
\hline 9 & Gentamycin & 0.05 & 1 & 0.25 & 0.5 \\
\hline 10 & Ampicillin & 100 & - & 250 & 100 \\
\hline 11 & Chloramphenicol & 50 & 50 & 50 & 50 \\
\hline 12 & Ciprofloxacin & 25 & 25 & 50 & 50 \\
\hline 13 & Norfloxain & 10 & 10 & 10 & 10 \\
\hline
\end{tabular}


Table 6. Antifungal activity study of $\mathbf{6 a}-\mathbf{e}$ with standard drugs.

\begin{tabular}{|l|l|l|l|l|}
\hline \multicolumn{5}{|c|}{ Minimal inhibition concentration $(\mu \mathrm{g} / \mathrm{mL})$} \\
\hline Entry & Compound & C. albicans $($ MTCC227) & A. niger $(\mathrm{MTCC} 282)$ & A. clavatus $($ MTCC1323) \\
\hline 1 & 6a & 1000 & $>1000$ & $>1000$ \\
\hline 2 & 6b & 500 & $>1000$ & $>1000$ \\
\hline 3 & 6c & 1000 & 1000 & 1000 \\
\hline 4 & 6d & 1000 & 500 & 500 \\
\hline 5 & 6e & 500 & $>1000$ & $>1000$ \\
\hline 9 & Nystatin & 100 & 100 & 100 \\
\hline 10 & Griseofulvin & 500 & 100 & 100 \\
\hline
\end{tabular}

\section{CONCLUSION}

The method of synthesis of $\mathbf{6 a - e}$ is aqueous phased, environmentally friendly and gives product easily in good yield without using any hazardous chemicals. Spectroscopic study of $6 \mathbf{6}-\mathbf{e}$ and Xray crystallographic study of $\mathbf{6 c}$ shows azo dye formation with tautemerism to retain aromaticity of isoxazole ring. All synthesized compounds showed antibacterial activity against $S$. aureus close to standard ampicillin. Compounds $\mathbf{6 c}$ and $\mathbf{6 e}$ showed antibacterial activity against $E$. coli close to ampicillin and $\mathbf{6 b}$ and $6 \mathrm{e}$ showed antifungal activity against $C$. albicans close to gresiofulvin. The crystal structure of $\mathbf{6 c}$ shows hydrogen bonding interaction with water molecule. Oxygen atom attached to isoxazole ring shows intermolecular as well as intramolecular hydrogen bonding.

\section{ACKNOWLEDGMENTS}

The authors are thankful to Microcare Laboratory and Tuberculosis Centre, Surat for providing antibacterial and antifungal activity. Financial support from the Slovenian Research Agency (ARRS) through project P1-0175 is gratefully acknowledged. We thank the EN-FIST Centre of Excellence, Ljubljana, Slovenia, for using SuperNova diffractometer.

\section{REFERENCES}

1. Dakiky, M.; Nemcova, I. Aggregation of O,O'-dihydroxy azo dyes III. Effect of cationic, anionic and non-ionic surfactants on the electronic spectra of 2-hydroxy-5-nitrophenylazo-4[3-methyl-1-(4"-sulfophenyl)-5-pyrazolone]. Dyes Pigm. 2000, 44, 181-193.

2. Navarro, A.; Sanz, F. Dye aggregation in solution: Study of C.I. direct red I. Dyes Pigm. 1999, 40, 131-139.

3. Tao, J.; Mao, G.; Daehne, L. Asymmetrical molecular aggregation in spherulitic dye films. J. Am. Chem. Soc. 1999, 121, 3475-3485.

4. Summers, L.A.; Freeman, P.F.H.; Shields, D.J. Structure of 3-alkyl-4-arylazoisoxazol-5ones and related compounds. J. Chem. Soc. 1965, 3312-3318.

5. Maradiya, H.R. Synthesis and dyeing performance of some novel thiazole azo disperse dyes. J. Saudi Chem. Soc. 2010, 14, 77-81.

6. Al-Hamdani, A.A.S.; Balkhi, A.M.; Falah, A.; Shayma, A.; Shaker, S.A. Synthesis and investigation of thermal properties of vanadyl complexes with azo-containing Schiff-base dyes. J. Saudi Chem. Soc. 2016, 20, 487-501.

7. Karci, F; Sener, I.; Deligoz, H. Azocalixarenes. 2: Synthesis, characterization and investigation of the absorption spectra of azocalix[6] arenes containing chromogenic groups. Dyes Pigm. 2004, 62, 131-140. 
8. Parkanyi, C.; Shawali, A.S. An HMO study of the azo-hydrazonetautomerism in diazonium coupling products of 5-isoxazolones. J. Heterocycl. Chem. 1980, 17, 897-903.

9. Iranpoor, N.; Firouzabadi, H.; Khalili, D. 5,5'-Dimethyl-3,3'-azoisoxazole as a new heterogeneous azo reagent for esterification of phenols and selective esterification of benzylic alcohols under Mitsunobu conditions. Org. Biomol. Chem. 2010, 8, 4436-4443.

10. Goda, F.E.; Maarouf, A.R.; El-Bendary, E.R. Synthesis and antimicrobial evolution of new isoxazol and pyrazole derivatives. Saudi Pharm. J. 2003, 11, 111-117.

11. Gouda, M.A.; Eldien, H.F.; Girges, M.M.; Berghot, M.A. Synthesis and antitumor evaluation of thiophene based azo dyes incorporating pyrazolone moiety. J. Saudi. Chem. Soc. 2016, 20, 151-157.

12. Goyal, R.N.; Jain, R.T.L.C. Separation of some closely related potential antibiotic 3,5dimethyl-4-arylazoisoxazoles. Fresen. Z. Anal. Chem. 1978, 292, 238-238.

13. Bertolasi, V.; Gilli, P.; Ferreti, V.; Gilli, G. Intramolecular N-H--O hydrogen bonding assisted by resonance. III.* Structural studies of 1-ketone-2-arylhydrazone derivative. Acta Crystallogr. Sect. B Struct. Sci. 1994, B50, 617-625.

14. Rageh, H.M.; Ibrahim, S.A.; Selim, M.A.; Alsoghier, H.M. Spectroscopic and semiempirical investigation of the structural features of hetarylazo-5-isoxazolonestautomerism. J. Saudi Chem. Soc. 2017, 21, S467-S474.

15. CrysAlis PRO, Agilent Technologies, Yarnton, England; 2013.

16. Palatinus, L.; Chapuis, G. SUPERFLIP - A computer program for the solution of crystal structures by charge flipping in arbitrary dimensions. J. Appl. Crystallogr. 2007, 40, 786790.

17. Sheldrick, G.M. SHELXT - Integrated space-group and crystal-structure determination Acta Cryst. 2015, C71, 3-8.

18. Isenberg, H.D. Clinical Microbiology Procedure Handbook, Vol. II, 2nd ed., American Society for Microbiology: Washington; 1998; chapter 5, p 501.

19. Desai, N.C.; Shihora, P.N.; Moradia, D.L. Synthesis and characterization of new quinazolines as potential antimicrobial agents. Indian J. Chem. 2007, 46B, 550-553.

20. National Committee for Clinical Laboratory Standards. Methods for Dilution, Antimicrobial Susceptibility Tests for Bacteria that Grow Aerobically Approved Standard, (M7A5), 5th ed., National Committee for Clinical Laboratory Standards: Wayne, PA, USA; 2000.

21. Shadomy S. in Manual of Clinical Microbiology, Albert, B. (Ed.), ASM Press: Washington, DC; 1991; p 1173.

22. Rattan A. Antimicrobials in Laboratory Medicine, BI Churchill Livingstone: India; 2000; p 85 .

23. Rehman, H.; Qadir, A.; Ali, Z.; Nazir, S.; Zahra, A.; Shahzady, T.G. Synthesis and characterization of novel sulfonamides derivatives and their antimicrobial, antioxidant and cytotoxicity evaluation. Bull. Chem. Soc. Ethiop. 2017, 31, 491-498.

24. Wazalwar, S.S.; Banpurkar, A.R.; Perdih, F. Aqueous phase synthesis, crystal structure and biological study of isoxazole extensions of pyrazole-4-carbaldehyde derivatives $\mathrm{J}$. Mol. Struct. 2017, 1150, 258-267. 